\title{
Ulk1/Rab9-mediated alternative mitophagy confers cardioprotection during energy stress
}

\author{
Rimpy Dhingra, ${ }^{1}$ Inna Rabinovich-Nikitin, ${ }^{1}$ and Lorrie A. Kirshenbaum ${ }^{1,2}$ \\ 'The Institute of Cardiovascular Sciences, St. Boniface Hospital Albrechtsen Research Centre, Department of Physiology and Pathophysiology, and Rady College of Medicine, Max Rady Faculty \\ of Health Sciences, University of Manitoba, Winnipeg, Manitoba, Canada.
}

\begin{abstract}
The heart relies on mitochondria-derived energy production for continuous contraction and relaxation; therefore, the maintenance of a pool of healthy mitochondria is essential for sustaining normal cardiac performance. Mitophagy serves as a critical process for maintaining mitochondrial quality control and involves the PTEN-induced kinase 1/Parkin (Pink1/ Parkin) pathway and autophagosomes labeled with the autophagy proteins autophagy-related 7 (ATC) and light chain 3 (LC3). In this issue of the JCI, Saito and colleagues identify an alternative pathway for mitophagy that utilizes the serine/threonine protein kinase Unc-51-like kinase 1 (Ulk1) and the small GTPase Rab9 to clear damaged mitochondria independently of conventional autophagy proteins. Together, the results of this study reveal that Ulk1 phosphorylation of Rab9 at serine 179 is critical for alternative mitophagy and cardioprotection under energy stress conditions.
\end{abstract}

\section{Conventional mitophagy}

Autophagy is a highly conserved physiological process by which intracellular macromolecules, organelles, and proteins are degraded by a lysosome-regulated pathway. As an adaptive response mechanism, autophagy affords the cell the ability to avert the cytotoxic effects of damaged organelles and aggregated proteins during cellular stress, such as nutrient deprivation or ischemia. During general autophagy, the cytoplasmic constituents that are targeted for destruction are sequestered in a phagophore that is derived from the endoplasmic reticular membrane. The phagophore then undergoes a series of maturation steps that include nucleation, elongation, and conjugation. These processes are mediated by the autophagy protein complexes Beclin1VPS34-VPS15 (Beclin1 is also known as ATG6), ATG12-ATG5, and ATG8-light chain 3 (ATG8-LC3), respectively, and lead to formation of the double-membraned autophagosome. The association of the autophagosome with adaptor proteins, such as LC3-p62, facilitates fusion with acid-rich lysosomes and results in degradation of the autophagosome. Hence, autophagy is a quality control mechanism for maintaining cellular homeostasis by eliminating toxic biomolecules (1-3).

Damaged mitochondria are removed by a specialized form of autophagy, referred to as mitophagy. Mitophagy is best known to be regulated by the PTEN-induced kinase 1/Parkin (Pink1/ Parkin) pathway (reviewed in refs. 4-6). The cellular kinase Pink1 and the ubiquitin E3-ligase Parkin were proposed to play a central role in mitochondrial clearance in Parkinson's disease, as mutations in the genes encoding Pink1, Parkin, or both have been found in patients with the disease (7). In this model, Parkin is retained in the cytoplasm but is phosphorylated and recruited to the outer membranes of damaged mitochondria following Pink1 stabilization. The Parkin-mediated polyubiquitination of outer mitochondrial membrane proteins targets depolarized mitochondria

Related Article: p. 802

Conflict of interest: The authors have declared that no conflict of interest exists.

Reference information: / Clin Invest. 2019;129(2):509-512. https://doi.org/10.1172/JCI125980. 


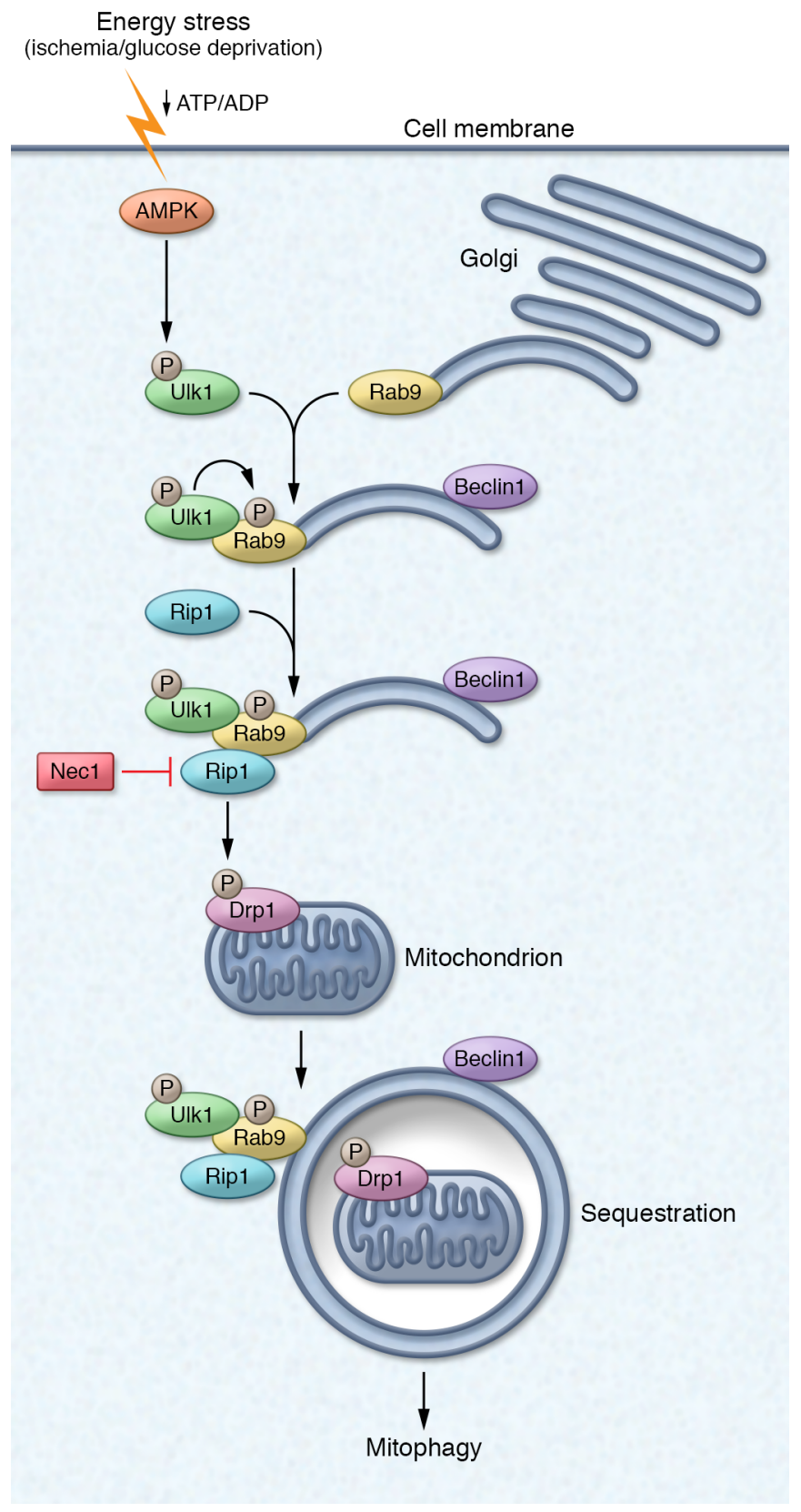

unclear (16). In this issue of the JCI, Saito et al. provide insight into the mechanisms that underlie alternative mitophagy in the heart imposed by energy stress (17). This study provides compelling evidence that Ulk1 phosphorylation of Rab9 at serine 179 is an essential step for the initiation of mitophagy under energy stress conditions. Through a variety of elegantly designed experiments, the authors show that mitophagy in cardiac myocytes involves phosphorylation of Rab9 by Ulk1, which then serves as a signaling platform for the formation of a complex between the receptorinteracting protein 1 (Rip1) kinase and dynamin-related protein (Drp1) (Figure 1).
To better understand the underlying mechanisms of mitophagy activation in the heart during energy stress, Saito et al. subjected WT and cardiac-specific Atg7knockout (Atg7-cKO) mice to 48 hours of glucose starvation (17). As predicted, LC3 levels and autophagy flux were increased in WT mice but not in Atg7-cKO mice. Surprisingly, despite low LC3 levels, electron micrographs of Atg7-cKO hearts displayed numerous mitochondria surrounded by double-membrane structures. Next, the authors generated Atg7-cKO/MitoKeima-Tg mice, which allow reliable evaluation of mitophagy, and subjected them to starvation to verify that mitochondria
Figure 1. Ulk1 phosphorylation of Rab9 at serine 179 is essential for mitophagy and cardioprotection during energy stress. In this issue of the $J C l$, a study by Saito et al. provides molecular insight into an alternative mitophagy pathway activated in the heart under energy stress conditions (17). Energy stress provoked by glucose deprivation or hypoxia/ischemia activates AMPK, which then phosphorylates Ulk1. Ulk1 forms a complex with the Rasrelated protein Rab9, which is associated with a Colgi-derived membrane. Ulk1 phosphorylates Rab9 at serine 179, and phosphorylated Rab9 then serves as a platform for the formation of a complex between Rip1 and Drp1. Rip1 phosphorylates Drp1 at serine 616 in a Ulk1- and Rab9-dependent manner. Mitochondria with phosphorylated Drp1 are recognized and sequestered by Rab9-associated membranes, followed by fusion with lysosomes and digestion. The Rip kinase inhibitor necrostain-1 (Nec1) inhibits energy stress-induced mitophagy.

surrounded by double membranes were actually sequestered within autophagosomes. Indeed, confocal and electron microscopic analyses revealed that starvation-induced mitophagy was independent of the conventional autophagy proteins Atg7 and LC3. Interestingly, the Atg7-cKo hearts displayed increased levels of Ulk1, which had been previously shown to trigger alternative mitophagy under different stress conditions (16). Saito et al. then explored the involvement of Ulk1 on mitochondrial clearance by testing the effects of starvation on mitophagy activation in mice with cardiac-restricted knockout of Ulk1 (Ulk1-cKO). Ulk1-cKO mice readily displayed increased levels of LC3 and preserved autophagy flux; however, despite the presence of an intact LC3 autophagy clearance system, Ulk1-cKO/Mito-KeimaTg mice exhibited a sharp decline in mitophagy and mitochondrial clearance. These findings strongly supported the contention that activation of mitophagy during starvation requires Ulk1 and but not Atg7-LC3.

Finally, Saito and colleagues explored the mechanisms by which Ulk1 activates mitophagy in cardiac myocytes. The authors began by investigating whether the autophagosomal membranes in the Ulk1 pathway are derived from trans-Golgi and endosomes and associated with Rab9 (17). Specifically, inhibition of Golgiderived membranes with brefeldin A resulted in a marked reduction in mitophagy, without any effect on convention- 
al autophagy markers. Moreover, Rab9, but not LC3, colocalized with autophagosomes and autolysosomes during energy stress, and knockdown of Rab9 disrupted energy stress-induced mitophagy. Interestingly, colocalization of Rab9 with autophagosomal or lysosomal membranes was highly dependent on Ulk1, and loss of Ulk1 or Rab9 impaired mitophagy and mitochondrial function during energy stress, demonstrating the importance of Ulk1 and Rab9 for mitophagy activation under these conditions. In addition, phosphorylation of Ulk1 by AMPK was critical for mitophagy during energy stress. As mitochondrial fission has previously been shown to be a requisite event for mitochondrial clearance by mitophagy, Saito et al. evaluated the contribution of the mitochondrial fission protein Drp1 in Ulk1/Rab9-mediated mitophagy. Indeed, Drp1 phosphorylation at serine 616 was increased and corresponded with increased mitochondrial fission during energy stress. Interestingly, Drp1 phosphorylation was contingent on Ulk1, as inhibition of Ulk1 in cardiomyocytes, or in Ulk1-cko mice, resulted in a marked decline in Drp1 phosphorylation. Interestingly, Rip1, a kinase typically involved in NF- $\mathrm{kB}$ signaling and necroptosis, directly phosphorylated Drp1 during energy stress, and mass spectroscopic analysis identified a protein complex composed of Ulk1, Rab9, Rip1, and Drp1. Further studies revealed that Ulk1 interaction and phosphorylation at serine 179 are critical for Rip1 association and phosphorylation of Drp1. Hence, mitochondria harboring phosphorylated Drp1 are sequestered and targeted by Rab9 for mitophagy. While alternative mitophagy by Ulk1 has been previously demonstrated, the activation of this pathway in the heart during energy stress, as described in this study, is relatively novel - especially the identification of Ulk1 as a critical kinase required for phosphorylation of Rab9 at serine 179 and the contribution of Drp1-mitochondrial fission for mitophagy activation and cardioprotection during energy stress.

\section{Conclusions and future directions}

While the study by Saito et al. highlights an alternative mode for mitophagy activation and mitochondrial clearance in cardiac myocytes, it also raises several issues for future consideration. For instance, what are the signals that direct mitochondrial clearance by conventional mitophagy mediated by Parkin/Atg8-LC3 or by the alternative Ulk1/Rab9 pathway? Perhaps alternative autophagy provides a fail-safelike mechanism to ensure that damaged mitochondria are removed at any cost in the event that the conventional pathway is blocked or inactivated. Alternatively, maybe the cells preferentially activate one pathway over the other on the basis of the type and severity of cellular stress. Another area of interest relates to how mitochondria that are undergoing Drp1-mediated fission become selectively removed by the alternative pathway, as Drp1-mediated fission is required for efficient mitochondrial clearance by the conventional pathway. At present, it is undetermined whether Ulk1/Rab9-mediated mitophagy is a universally conserved feature that serves as a mitophagy back-up system or is activated in response to other stress conditions, such as pressure-overload hypertrophy, inflammation, and/or drug-induced heart failure. Finally, what is the importance of the Ulk1/Rab9 mitophagy pathway in other organs, such as lung and brain, that may differ in mitochondrial abundance compared with cardiac myocytes and may therefore utilize different modes of autophagy for mitochondrial quality control?

Moreover, Rip1, which is involved in the regulation of necroptosis among other signaling pathways including that for $\mathrm{NF}-\mathrm{KB}$ activation, was shown in the present study to interact with Rab9 in close proximity with mitochondria. However, it is unclear how Rip1, which otherwise mediates necroptosis signaling through associations with Rip3 and mixed-lineage kinase domain-like pseudokinase (MLKL), interacts with Rab9 to execute mitophagy. Generally, Rip1 dissociates from receptors in the necrosome in response to death receptor activation and necrosis signaling, but in the Saito et al. study, Rip1 was shown to be a part of the Rab9 alternative mitophagy pathway, which was cardioprotective (17). A better understanding of how Rip1 is regulated during cellular stress may provide important insight into how necrosis and mitophagy can be differentially influenced by Rip1. Nevertheless, the study by Saito et al. has revealed a novel signaling pathway for mitophagy in cardi- ac myocytes that coordinates overlapping pathways between Golgi-endosomal trafficking and necroptosis pathways via the formation of Ulk1-Rab9-Rip1 complexes for cardioprotection.

\section{Acknowledgments}

This work was supported by a foundation grant to LAK from the Canadian Institute for Health Research (CIHR) and the St. Boniface Hospital Foundation. LAK holds a Canada Research Chair in Molecular Cardiology. IRN holds a postdoctoral fellowship from the CIHR.

Address correspondence to: Lorrie A. Kirshenbaum, Institute of Cardiovascular Sciences, St. Boniface Hospital Albrechtsen Research Centre, Rm. 3016, 351 Taché Avenue, Winnipeg, Manitoba, Canada, R2H 2A6. Phone: 204.235.3661; Email: Lorrie@sbrc.ca.

1. Glick D, Barth S, Macleod KF. Autophagy: cellular and molecular mechanisms. J Pathol. 2010;221(1):3-12.

2. Biala AK, Kirshenbaum LA. The interplay between cell death signaling pathways in the heart. Trends Cardiovasc Med.2014;24(8):325-331.

3. Sciarretta S, Maejima Y, Zablocki D, Sadoshima $\mathrm{J}$. The role of autophagy in the heart. Annu Rev Physiol. 2018;80:1-26.

4. Dorn GW 2nd. Parkin-dependent mitophagy in the heart. J Mol Cell Cardiol. 2016;95:42-49.

5. Dorn GW 2nd. Central Parkin: The evolving role of Parkin in the heart. Biochim Biophys Acta. 2016;1857(8):1307-1312.

6. Dorn GW 2nd. Jurassic PARK2: You eat your mitochondria, and you are what your mitochondria eat. Autophagy. 2016;12(3):610-611.

7. Pickrell AM, Youle RJ. The roles of PINK1, parkin, and mitochondrial fidelity in Parkinson's disease. Neuron. 2015;85(2):257-273.

8. Narendra DP, et al. PINK1 is selectively stabilized on impaired mitochondria to activate Parkin. PLoS Biol. 2010;8(1):e1000298.

9. Chen Y, Dorn GW. PINK1-phosphorylated mitofusin 2 is a Parkin receptor for culling damaged mitochondria. Science. 2013;340(6131):471-475

10. Moyzis AG, Sadoshima J, Gustafsson ÅB. Mending a broken heart: the role of mitophagy in cardioprotection. Am J Physiol Heart Circ Physiol. 2015;308(3):H183-H192.

11. Hoshino A, et al. Cytosolic p53 inhibits Parkinmediated mitophagy and promotes mitochondrial dysfunction in the mouse heart. Nat Commun. 2013;4:2308.

12. Kubli DA, et al. Parkin protein deficiency exacerbates cardiac injury and reduces survival following myocardial infarction. J Biol Chem. 2013;288(2):915-926.

13. Lee Y, Lee HY, Hanna RA, Gustafsson ÅB. Mitochondrial autophagy by Bnip3 involves Drp1mediated mitochondrial fission and recruitment 
of Parkin in cardiac myocytes. Am J Physiol Heart Circ Physiol. 2011;301(5):H1924-H1931.

14. Billia F, Hauck L, Konecny F, Rao V, Shen J, Mak TW. PTEN-inducible kinase 1 (PINK1)/Park6 is indispensable for normal heart function. Proc Natl Acad Sci U S A.
2011;108(23):9572-9577.

15. Maejima Y, Chen Y, Isobe M, Gustafsson ÅB, Kitsis RN, Sadoshima J. Recent progress in research on molecular mechanisms of autophagy in the heart. Am J Physiol Heart Circ Physiol. 2015;308(4):H259-H268.
16. Nishida Y, et al. Discovery of Atg5/Atg7-independent alternative macroautophagy. Nature. 2009;461(7264):654-658.

17. Saito T, et al. An alternative mitophagy pathway mediated by Rab9 protects the heart against ischemia. J Clin Invest. 2019;129(2):802-819. 